\title{
Bioorthogonal Chemistry in Biology and Medicine
}

Chakraborty $\mathbf{P}^{*}$

Indian Institute of Chemical Biology, Kalpana Chawla Center for Space and Nanosciences, Kolkata, India

\begin{abstract}
Bioorthogonal chemistry and reactions developed from innovations in chemistry, having their excellent biocompatibility can be applied in various biological reactions. As these reactions, does not interfere with other reactions in the biological system, they can be used as excellent tools for tracking and studying dynamics of various biological processes. In this mini review, only the applications of bioorthogonal chemistry in host-microbe interaction and targeted therapy are discussed.
\end{abstract}

Keywords: Bioorthogonal chemistry; Bioorthogonal reaction; Biomolecules; Microbes; Host; Gene delivery

\section{Introduction}

Bioorthogonal chemistry, developed from innovations in chemistry, refers to any chemical reaction that can occur inside living system without interfering with native biochemical processes. Since the introduction of bioorthogonal reaction, it has been applied to diverse classes of biomolecules like glycans, lipids, proteins, nucleic acids of the cells, with excellent biocompatibility and without cellular toxicity $[1,2]$. Bioorthogonal reactions which fulfill the requirements of bioorthogonality are i) 1,3-dipolar cycloaddition between azides and cyclooctynes, ii) nitrones and cyclo-octynes, iii) tetrazine ligation and iv) oxime/hydrazone formation from aldehydes and ketones (Figures $1 \mathrm{~A}$ and $1 \mathrm{~B})$. The classic azide-alkyne cyclo-addition reaction very effective click reaction and ideal for bioconjugation. These reactions take part in biological system as, first, biomolecules(substrate) labelled with bioorthogonal functional group (like azides) introduced into the cell and then a probe containing complementary functional group (like alkynes) introduced into the cell to react and label the substrate (Figure 2).

\section{Literature Review}

Before, bioorthogonal reactions, GFPs (green-fluorescent proteins) were used for the study of protein dynamics and functions in biological systems, however this genetic tagging approach were not suitable for the studies involving other biomolecules such as glycans, lipids, nucleic acids and post-translational modification reactions in biological cells. Since the first appearance of biorthogonal chemistry in 2003, although there are some comprehensive reviews [3-5] on developments of various biorthogonal reactions and applications, no single review todate which focuses on applications of biorthogonal reactions in host-microbe interaction and in targeted therapy. As we were engaged in cell-cell interactions [6-8], and drug targeting in leishmaniasis [9-11], we are planning to apply the biorthogonal reactions in emerging pathogen interaction with the host. It is hoped, that this will be a challenging area for future reseach to understand the mechanism of survival of these emerging intracellular pathogens around the world.

\section{Bioorthogonal chemistry in host-microbe interaction}

Suitable receptor-ligand interaction between host and microbe helps to cross the microbe the host cell surface and gain entry inside the host cell. Intracellular localization places the microbe in an environment which is potentially rich in nutrients and devoid of competing microorganisms. However, the intracellular life for these pathogens is not very easy as they have to face or escape the host cell acidic environment or potentially degradative lysosomes. Different intracellular pathogens exploit different locations of the host cells and changeable strategies to sustain their infection [8].

The essential components of microbe cell surfaces are peptidoglycan, lipoarabinomannan, lipophosphoglycan, lipopolysaccharides etc. and these molecules are responsible for pathogen-host interaction and intracellular survival of the pathogens $[12,13]$. Till date little is known about the synthesis, maturity and dynamics of these pathogen molecules when they are in the intracellular environment, however, there is lots of information when they are outside. Efforts to track synthesis, editing and dynamics of these molecules in the intracellular environment suffer from several drawbacks. These includes species applicability, technical complexity etc.. Recent work on tracking synthesis and maturation of peptidoglycan molecule of bacteria in the intracellular environment in Listeria monocytogenes-macrophage model involves bioorthogonal chemistry/bond-formation reaction and metabolic labelling [14]. The technology was the exploitation of D-alanine constituents of peptidoglycan of bacteria, and the bacterial species incorporated azide or alkyne-functionalized D-alanine into their cell walls which was visualized by covalent reaction with click chemistry probes (Figure 2). As eukaryotic cells do not generally produce $\mathrm{D}$-amino acids, this labelling was selectively used for bacteria inside the host cell, and other recent study also demonstrated that this labelling technology could also be used for wide variety of bacterial species. If this technology, could be extended to other glycoconjugates and other microbes then it would be ideal for the study of mechanism of intracellular survival of wide variety of microbes and emerging pathogens.

\section{Bioorthogonal chemistry in targeted therapy}

To reduce normal cell toxicity/tissue injury from cytotoxic drugs, targeted therapy is the solution to cure the diseased/infected cells. Targeted therapy involves gene therapy or drug targeting against various critical diseases. Gene therapy have utilized viral-based delivery systems, and in recent years, gene manipulation and delivery

*Corresponding author: Dr. Prasanta Chakraborty, Retired Senior Scientist Indian Institute of Chemical Biology, Kalpana Chawla Center for Space and Nanosciences, Akashdeep, 184, Santoshpur Avenue, Kolkata- 700075, India, Tel: 91332416 7786; E-mail: prasanta3274@yahoo.in

Received February 12, 2018; Accepted March 02 2018; Published March 05 2018

Citation: Chakraborty P (2018) Bioorthogonal Chemistry in Biology and Medicine. J Mol Genet Med 12: 332 doi:10.4172/1747-0862.1000332

Copyright: ( 2018 Chakraborty P. This is an open-access article distributed under the terms of the Creative Commons Attribution License, which permits unrestricted use, distribution, and reproduction in any medium, provided the original author and source are credited 
A.

ii)<smiles>Cc1n[n-]c(C)n1</smiles>

iii)

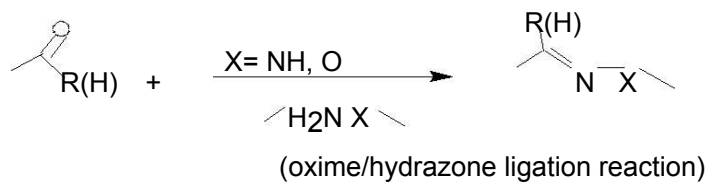

B.

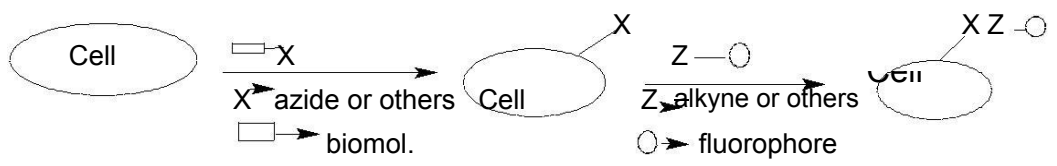

Figure 1: i), ii), iii) Bioorthogonal reactions. B. Azide or other bioorthogonal functional group labelled biomolecules is introduced into the cell. Then cyclooctynes or another complementary functional group containing probe is introduced into the cell to react and label the biomolecule.
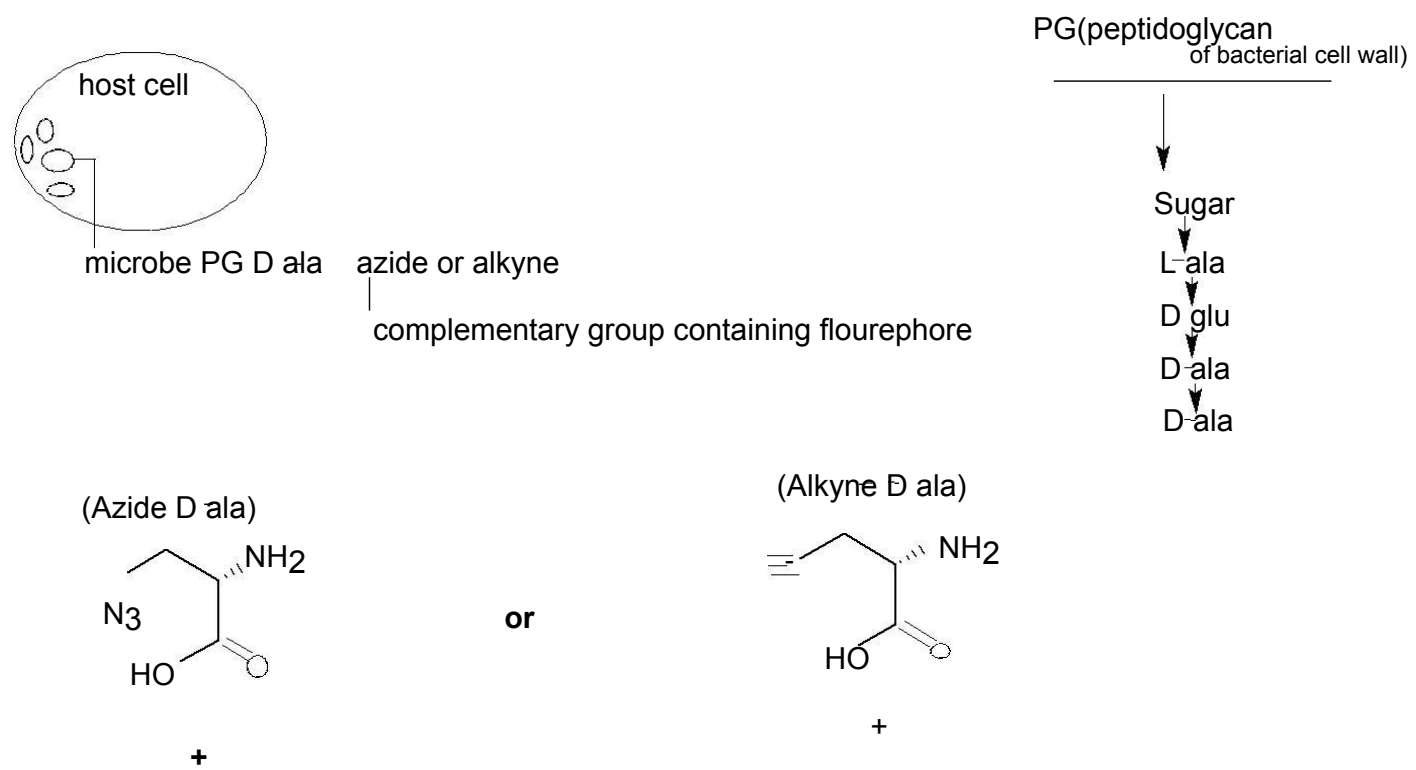

(Alkyne $\mathrm{B}$ ala)

complementary group containing fluorephore<smiles>C#CC[C@H](N)C(=O)O</smiles>

complementary group containing fluorephore

Figure 2: Bioorthogonal reaction in host microbe interaction. Bacterial species inside host cell incorporated azide or alkyne functionalized D alanine into PG of their cell walls and visualized by covalent reaction with click chemistry probes. 


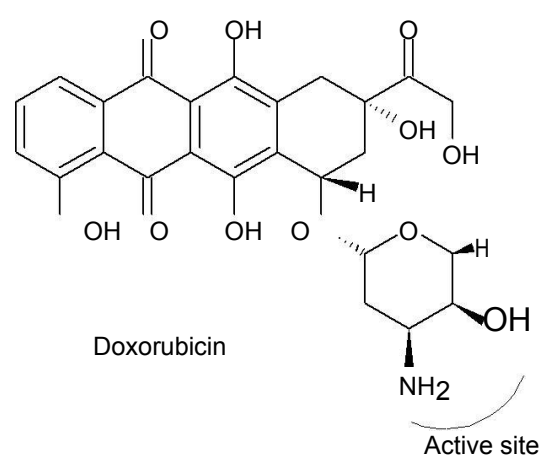<smiles>CCCCC</smiles>

Ru_catalyzed decaging inside HeLa cell<smiles>[2H][C@@]1(CO)CC(C(=O)CC)CC(=C)c2c(O)c3c(c(C)c21)C(=O)c1c(O)cccc1C3=O</smiles>

Figure 3: Seletive liberation of potent cytotoxic drugs via bioorthogonal cleavage reactions. Chemically caged doxorubicin was deprotected through bond cleavage reagents. Ruthenium(Ruf catalyzed deallylation reaction was used to activate alloxycarbonyl protected doxorubicin in HeLa cells.

such as CRISPR and non-viral vectors (e.g. liposomes) have been used against hemophilia B, Parkinson's disease, acute lymphocytic leukemia, multiple myeloma, HIV [15,16] whereas drug targeting using neoglycoprotein, antibody and liposome as carriers have been targeted against various infectious diseases [9-11]. However, there are various difficulties/disadvantages associated with these therapies, like inflammatory response of viral vectors, vector viruses infecting more than one type of cell, insertion of a new gene in the wrong location, toxicity etc. Bioorthogonal chemistry here plays a vital role in search of a new system to target drugs to the diseased site specifically. For example, bioorthogonal cleavage reactions have recently been used for selective liberation of potent cytotoxic drugs in a spatiotemporally controlled manner $[17,18]$. The reaction, Ruthenium $(\mathrm{Ru})$-catalyzed deallylation was used to activate alloxycarbonyl(Alloc)-protected doxorubicin in HeLa cells (Figure 3).

\section{Discussion and Conclusion}

The study of biomolecules in their native environment is a challenging task, because of the huge complexity of the cellular system. In the last few years, among the new technologies developed for studying the modification of biomolecules and their dynamics inside cells are the biorthogonal bond formation and bond cleavage reactions. Non-interference of these reactions with the other biological reactions in the system indicates their great promise in future. Continuous development of further reactions for various kinds of biological cells and tissues would definitely set these reactions as new tools for biology and medicine.

\section{References}

1. Li J, Chen PR (2016) Development and application of bond cleavage reactions in bioorthogonal chemistry. Nat Chem Biol 12: 129-137.

2. Ramil CP, Lin Q (2013) Bioorthogonal chemistry: Strategies and recent developments. Chem Commun 49: 11007-11022.

3. Mckay CS, Finn MG (2014) Click chemistry in complex mixtures: Bioorthogonal bioconjugation. Chem biol. 21: 1075-1101.

4. Zheng M, Zheng L, Zhang P, Jinbo L, Zhang $Y$ (2015) Development of biorthogonal reactions and eir applications in bioconjugation. Molecules 20: 3190-3205.

5. Grammel M, Hang HC (2013) Chemical reporters for biological discovery. Nat Chem Biol 9: 475-484.

6. Chakraborty P, Das PK (1988) Role of mannose/N-acetyl glucosamine receptors in blood clearance and cellular attachment of Leishmania donovani. Mol Biochem Parasitol 28: 55-62.

7. Chakraborty P (2001) G-protein mediated signaling and its control in macrophages and mammalian cells. Crit Rev Microbiol 27: 1-8.

8. Chakraborty P, Koszycki SS, Russell DG (1994) Isolation and characterization of pathogen containing Phagosome. Meth Cell Biol 45: 261-274.

9. Chakraborty P, Bhaduri AN, Das PK (1990) Sugar receptor mediated drug delivery to macrophages in the therapy of experimental visceral leishmaniasis. Biochem Biophys Res Commun 166: 404-410.

10. Chakraborty P, Bhaduri AN, Das PK (1990) Neoglycoprotein as carriers for receptor mediated drug targeting in the treatment of experimental visceral leishmaniasis. J Protozool 37: 358-364.

11. Chakraborty P, Basu MK (1997) Leishmania phagolysosome: Drug trafficking and protein sorting across the compartment. Crit Rev Microbiol 23: 253-260.

12. Mishra AK, Driessen NN, Appelmelk BJ, Besra GS (2011) Lipoarabinomannan and related glycoconjugates: structure,biogenesis and role in Mycobacterium tuberculosis physiology and host-pathogen interaction. FEMS Microbiol Rev 35: 1126-1157.

13. Mcneely TB, Turco SJ (1990) Requirement of lipophosphoglycan for intracellular survival of Leishmania donovani. J Immunol 144: 2745-2750.

14. Siegrist MS, Sarah W, Jewett JC, Aditham A, Cava F, et al. (2013) d-amino acid chemical reporters reveal peptidoglycan dynamics of an intracellular pathogen. ACS Chem Biol 8: 500-505.

15. Beitelshees M, Hill A, Rostami P (2017) Pressing diseases that represent promising targets for gene therapy. Disc Med 24: 134-136.

16. Stone D, Niyonzima N, Jerome KR (2016) Genome editing and the next generation of antiviral therapy. Human genetics 135: 1071-1082.

17. Weiss JT, Dawson JC, Fraser C, Rybski W, Torres-Sanchez C, et al. (2014) Development and bioorthogonal activation of palladium-labile prodrugs of gemcitabine. J Med Chem 57: 5395-5404.

18. Weiss JT, Dawson JC, Macleod KG, Rybski W, Fraser C, et al. (2014) Extracellular palladium-catalysed dealkylation of 5-fluoro-l-propargyl-uracil as a bioorthogonally activated prodrug approach. Nat Commun 5: 3277-3286. 